\title{
Development and implementation of the exact exchange method for semiconductors using a localized basis set
}

\author{
J.J. Fernández ，C. Tablero, P. Wahnón
}

\begin{abstract}
One of the major deficiencies of density functional theory is presented in the approximation of the exchange energy term. An important advance in solving this problem has been the development of orbital-dependent exchange functionals. The exact exchange method is one of the best defined releases of such functionals. Up to now it has been applied in solid systems only using a plane wave representation basis set. In this paper we present a development and implementation of the exact exchange formalism for solid semiconductors using a basis set of localized numerical functions. The implementation of the exact exchange scheme has been carried out in the SIESTA code, as a new path to get the exchange part in the Kohn-Sham energy and potential. This program is an ab initio periodic fully self-consistent density functional code which uses norm-conserving non-local pseudopotentials. Linear combination of confined numerical pseudoatomic orbitals have been used to represent the Kohn-Sham orbitals. The calculation results of the electronic properties of several semiconductor systems using different qualities of the basis set are compared with experimental results and presented in this paper.
\end{abstract}

Keywords: Density functional theory; Exact exchange method; Exchange potential; Semiconductors properties

\section{Introduction}

Density functional theory (DFT) $[1-3]$ is nowadays one of the most popular techniques used for the study of the properties of materials due to its low computational workload. The formalism is, in principle, exact but its application to the calculation of the total energy of interacting systems requires approximations for the exchange and correlation energy.
Kohn and Sham (KS) [3] in their early works, proposed an approximation of the exchange and the correlation part of the total energy using a functional relationship between the density and the exchange-correlation energy based on the study of an homogeneous electron gas. This functional is known as the local density approximation (LDA) exchange-correlation functional.

To overcome the lack of non-locality in this functional form, a generalized gradient correction (GGA) in the density of the LDA has been widely used for studying many electronic systems and their properties [4-7]. 
Although the application of LDA and GGA approximations to inhomogeneous solid state systems has been remarkably successful in describing ground state properties and the dispersion of upper valence bands, there still remain some characteristic deficiencies. In particular, in semiconductors, there is an underestimation of the energy gap between the occupied and empty bands and an incorrect asymptotic behaviour of the exchange and correlation potential.

Several schemes beyond these approximations have been developed. The optimised effective potential (OEP) method [30] was originally applied successfully to spherical finite systems [8-24] and also in molecules for which the HOMO-LUMO energy differences as well as ionization potentials were significantly improved [25]. The use of this method has recently been generalized to the case of infinite extended periodic solids. In this approach, the functional of the energy contains an exact expression for the exchange energy and the same LDA or GGA approximation for the correlation energy term.

Within this scheme, several authors have proposed the use of an orbital-dependent functional for the exchange, based on the OEP method. One of best developments is the exact exchange method (EXX) [8-15]. In this method the authors make the conditions established in the method developed by Krieger, Li and Iafrate (KLI method) in late 90s more flexible [16-19]. They also generalized the method used by Kotani et al. [20-23] allowing this exchange functional to be studied within the framework of a non-spherical assumption in the form of the potential in any part of the space. Although this EXX formalism is quite general, the applications of the method in solids have been carried out mainly using a plane wave basis set [14].

In last few years, DFT theory, using the typical LDA and GGA approximations for the exchange term, has been used as a tool for studying the electronic properties of different kind of materials. In particular, these methods have been used recently to identify, at an atomic level, new semiconductor materials for high efficiency photovoltaic devices as well as studying all the properties of these materials [26-28].
The use of orbital-dependent functionals allows the electronic structure of the many-electron systems to be described accurately. Therefore, in order to have a deeper knowledge of the optoelectronic properties of these kinds of materials, it is necessary to implement a new orbital-dependent functional. The EXX method seems to be the best choice for our purposes.

In this work we present an implementation of the EXX method for solids [14,15] within a numerical localised basis set framework. This method is applied to the study of the properties of some semiconductor materials. The calculations presented here try to represent a benchmark for the implementation of the EXX method in order to use it in the determination of properties for new materials.

For this purpose we have developed, tested and implemented a new EXX functional scheme within the First Principles SIESTA [29] code, using the EXX method as a new path for obtaining the exchange part in the Kohn-Sham energy and potential.

The SIESTA program is a very efficient numerical DFT code that solves self-consistently the Kohn-Sham equations for solids and molecules within the local density or in the generalized gradient approximations of DFT. It combines the use of a localised, LCAO, basis set for the description of valence electrons and norm conserving nonlocal pseudopotentials for the atomic core. This choice of code has been made based on the great flexibility it has in the choice of the basis set used to represent the Kohn-Sham orbitals. The EXX exchange potential has been added as a set of routines that work interactively with the SIESTA code. We have chosen a basis set of Slater functions in order to represent the exchange density, which is then integrated in order to obtain the potential.

In the next section, we introduce the theoretical development of the orbital-dependent exchange functionals and the different types of approximations.

In Section 3 we present the methodology of implementing the EXX method within the SIESTA code and in Section 4 the computational details of this implementation. 
In Section 5 we present some applications of the method. In particular, we show the calculations obtained for the properties of several semiconductors, especially in trying to describe the correct band gap and bandwidth values. Finally in Section 6 we present the conclusions.

\section{Theoretical development of the orbital-dependent functionals}

The DFT theory proposes a solution to the many electron problem based on the Kohn-Sham equations [1-3]

$\left[-\frac{\hbar^{2}}{2 m} \nabla^{2}+v_{\text {eff }}(\mathbf{r})\right] \psi_{i}(\mathbf{r})=\varepsilon_{i} \psi_{i}(\mathbf{r})$

where $\varepsilon_{i}$ is a Lagrange multiplier ensuring the normalization in the wavefunctions, $\psi_{i}(\mathbf{r})$. The effective one electron potential,

$v_{\mathrm{eff}}(\mathbf{r})=v(\mathbf{r})+e^{2} \int \frac{n\left(\mathbf{r}^{\prime}\right)}{\left|\mathbf{r}-\mathbf{r}^{\prime}\right|} \mathrm{d} \mathbf{r}^{\prime}+v_{\mathrm{xc}}(\mathbf{r})$

contains the external, Hartree and exchange-correlation terms respectively. The exchange-correlation term is defined as

$v_{\mathrm{xc}}(\mathbf{r})=\frac{\delta E_{\mathrm{xc}}}{\delta n(\mathbf{r})}$

where $n(\mathbf{r})$ is the electronic density.

$v_{\mathrm{xc}}(\mathbf{r})$ is called the exchange-correlation potential, which is usually split into the exchange and the correlation terms

$v_{\mathrm{xc}}(\mathbf{r})=v_{x}(\mathbf{r})+v_{c}(\mathbf{r})$

In the DFT formalism the exchange-correlation term has to be approximated by some functional form. In their early work, Kohn and Sham proposed the local density approximation (LDA) $[2,3]$.

In this approximation the exchange-correlation energy is obtained by the functional relationship:

$E_{\mathrm{xc}} \approx E_{\mathrm{xc}}^{\mathrm{LDA}}=\int n(\mathbf{r}) \varepsilon_{\mathrm{xc}}^{\text {homo }}(n(\mathbf{r})) \mathrm{d} \mathbf{r}$

in which $\varepsilon_{\mathrm{xc}}^{\text {homo }}$ is the exchange-correlation energy per particle in the homogeneous (spatial) electron gas. The homogeneous electron gas is a fictional system made up of a set of uniformly spatially distributed electrons within a positive background of charge that compensates the electronic charge. The functional derivative of this quantity with respect to the density is the definition of the local density approximation to the exchange potential,

$v_{\mathrm{xc}}^{\mathrm{LDA}}(\mathbf{r}) \equiv \frac{\delta E_{\mathrm{xc}}^{\mathrm{LDA}}}{\delta n(\mathbf{r})}=\left.\frac{\mathrm{d}}{\mathrm{d} n}\left(n \varepsilon_{\mathrm{xc}}^{\mathrm{homo}}(n)\right)\right|_{n=n(\mathbf{r})}$

Equations (1)-(6) present a well-defined scheme in which the ground state density can be obtained for any electronic system.

However, this scheme has several deficiencies when used to study real electronic systems. One important deficiency is due to the approximation of the exchange potential. In order to overcome this deficiency new functional forms have been proposed [4-7]. These new forms (GGA) are based on the use of some functionals depending on both the density and its gradient:

$E_{\mathrm{xc}}^{\mathrm{GGA}}[n]=\int \mathrm{d} \mathbf{r} n(\mathbf{r}) \varepsilon_{\mathrm{xc}}(n(\mathbf{r})|, \nabla n(\mathbf{r})|)$

In last few years, some authors have proposed the use of a functional relationship between the exchange energy and the orbitals [8-25]. This particular approximation has improved the quality of the DFT results for electronic calculations.

There are several types of approximations when an orbital-dependent exchange functional is used. One of them is the method developed by Krieger, $\mathrm{Li}$ and Iafrate, the KLI method [16-19], and another one is the exact exchange method, EXX method, which has been developed for spherical systems by Kotani [20-23], and in a more general way by Görling and Levy [8-15]. All these methods are based on solving the optimized effective potential equation [30], also called the OEP equation, but with different ways of finding the solution. We are going to compare very briefly the most important properties of the KLI and the EXX methods.

The KLI method provides a solution to the OEP integral equation in terms of an approximate form of the Green function thus 


$$
\begin{aligned}
& \sum_{i=1}^{N} \int \mathrm{d} \mathbf{r}^{\prime}\left(v_{\mathrm{xc}}^{\mathrm{OEP}}\left(\mathbf{r}^{\prime}\right)-u_{\mathrm{xci}}\left(\mathbf{r}^{\prime}\right)\right) G_{s i}\left(\mathbf{r}, \mathbf{r}^{\prime}\right) \psi_{i}(\mathbf{r}) \psi_{i}\left(\mathbf{r}^{\prime}\right) \\
& \quad+\text { c.c. }=0
\end{aligned}
$$

where,

$u_{\mathrm{xc} i}(\mathbf{r})=\frac{1}{\psi_{i}(\mathbf{r})} \frac{\delta E_{\mathrm{xc}}^{\mathrm{OEP}}\left[\psi_{j}\right]}{\delta \psi_{i}(\mathbf{r})}$

and,

$G_{i i}\left(\mathbf{r}, \mathbf{r}^{\prime}\right)=\sum_{s=1, s \neq i} \frac{\psi_{s}(\mathbf{r}) \psi_{s}^{*}\left(\mathbf{r}^{\prime}\right)}{\varepsilon_{i}-\varepsilon_{s}}$

in which the energy difference in the denominator of Eq. (10) is replaced by a constant.

$G_{s i}\left(\mathbf{r}, \mathbf{r}^{\prime}\right) \simeq \frac{1}{\Delta \varepsilon}\left(\delta\left(\mathbf{r}-\mathbf{r}^{\prime}\right)-\psi_{i}(\mathbf{r})-\psi_{i}\left(\mathbf{r}^{\prime}\right)\right)$

If this approximation is introduced into the OEP integral equation, the exchange-correlation potential for the KLI equation is obtained,

$v_{\mathrm{xc}}^{\mathrm{KLI}}(\mathbf{r})=\frac{1}{2 n(\mathbf{r})} \sum_{i=1}^{N}\left|\psi_{i}(\mathbf{r})\right|^{2}\left[u_{\mathrm{xci} i}(\mathbf{r})+\left(\hat{v}_{\mathrm{xc}}^{\mathrm{KLI}}-\hat{u}_{\mathrm{xc} i}\right)\right]$

in which $\hat{v}_{\mathrm{xc}}^{\mathrm{KLI}}$ and $\hat{u}_{\mathrm{xc}}$ are the averaged values of $u_{\mathrm{xc}}(\mathbf{r})$ and $v_{\mathrm{xc}}^{\mathrm{KLI}}(\mathbf{r})$.

The solution of this equation is,

$\hat{v}_{\mathrm{xcj}}^{\mathrm{KLI}}=\hat{v}_{\mathrm{xcj}}^{S}+\sum_{i=1}^{N-1} N_{j i}\left(v_{\mathrm{xci}}^{\mathrm{KLI}}-\frac{1}{2}\left(\hat{u}_{\mathrm{xc} i}-\hat{u}_{\mathrm{xci}}^{*}\right)\right)$

where

$\hat{v}_{\mathrm{xcj} j}^{S}=\int \mathrm{d} \mathbf{r} \frac{\left|\psi_{j}(\mathbf{r})\right|^{2}}{n(\mathbf{r})} \sum_{i=1}^{N}\left|\psi_{i}(\mathbf{r})\right|^{2} \frac{1}{2}\left(u_{\mathrm{xci} i}(\mathbf{r})-u_{\mathrm{xci} i}^{*}(\mathbf{r})\right)$

In this equation $n(\mathbf{r})$ is the total electronic density, and $N_{j i}$ in Eq. (13) is

$N_{j i}=\int \mathrm{d} \mathbf{r} \frac{\left|\psi_{i}(\mathbf{r})\right|^{2}\left|\psi_{j}(\mathbf{r})\right|^{2}}{n(\mathbf{r})}$

The second way of solving the OEP integral equation is based on the use of the chain rule to expand the functional derivative for the exchange energy in terms of the density [8-15]. This derivative can be written as follows:

$$
\begin{aligned}
v_{x}(\mathbf{r})= & \frac{\delta E_{x}[n]}{\delta n(\mathbf{r})} \\
= & \sum_{v} \sum_{\mathbf{k}} \int \mathrm{d} \mathbf{r}^{\prime} \\
& \times \int \mathrm{d} \mathbf{r}^{\prime \prime}\left[\frac{\delta E_{x}[n]}{\delta \psi_{v \mathbf{k}}\left(\mathbf{r}^{\prime}\right)} \frac{\delta \psi_{v \mathbf{k}}\left(\mathbf{r}^{\prime}\right)}{\delta v_{\mathrm{KS}}\left(\mathbf{r}^{\prime \prime}\right)}+\text { c.c. }\right] \frac{\delta v_{\mathrm{KS}}\left(\mathbf{r}^{\prime \prime}\right)}{\delta n(\mathbf{r})}
\end{aligned}
$$

where the $\psi_{v \mathbf{k}}(\mathbf{r}) \mathrm{s}$ are used to represent the different Kohn-Sham orbitals, $v_{\mathrm{Ks}}(\mathbf{r})$ represents the KohnSham potential, $v$ names the occupied monoelectronic states and $\mathbf{k}$ represents the translational quantum number of the wave functions, $\psi_{v \mathbf{k}}(\mathbf{r})$. Within this formalism, it is possible to build up an exchange density as a combination of the different localized functions [13].

$\rho_{x}(\mathbf{r})=\sum_{j} \alpha_{j} f_{j}(\mathbf{r})$

where the $\alpha_{j}$ s are the coefficients for the expansion. Then the integral of this density to obtain the exchange potential is carried out by

$v_{x}(\mathbf{r})=\sum_{j} \alpha_{j} \int \frac{f_{j}\left(\mathbf{r}^{\prime}\right)}{\left|\mathbf{r}-\mathbf{r}^{\prime}\right|} \mathrm{d} \mathbf{r}^{\prime}$

Using this scheme, the exchange potential has the correct asymptotic $(1 / r)$ behaviour.

In order to obtain the expansion coefficients shown in Eqs. (17) and (18) it is necessary to represent the linear response operator $X\left(\mathbf{r}, \mathbf{r}^{\prime}\right)$ on the basis set made up of the $f_{j}(\mathbf{r})$ functions.

$$
\begin{aligned}
X_{i j}= & \int \mathrm{d} \mathbf{r}_{1} f_{i}\left(\mathbf{r}_{1}\right) \int \frac{\mathrm{d} \mathbf{r}_{2}}{\left|\mathbf{r}_{1}-\mathbf{r}_{2}\right|} \int \mathrm{d} \mathbf{r}_{3} X\left(\mathbf{r}_{2}, \mathbf{r}_{3}\right) \\
& \times \int \mathrm{d} \mathbf{r}_{4} \frac{f_{j}\left(\mathbf{r}_{4}\right)}{\left|\mathbf{r}_{3}-\mathbf{r}_{4}\right|}
\end{aligned}
$$

where,

$$
\begin{aligned}
X\left(\mathbf{r}, \mathbf{r}^{\prime}\right)= & 2 \sum_{v} \sum_{c} \sum_{\mathbf{k}} \\
& \times \frac{\psi_{v \mathbf{k}}^{*}(\mathbf{r}) \psi_{c \mathbf{k}}(\mathbf{r}) \psi_{c \mathbf{k}}^{*}\left(\mathbf{r}^{\prime}\right) \psi_{v \mathbf{k}}\left(\mathbf{r}^{\prime}\right)}{\varepsilon_{v \mathbf{k}}-\varepsilon_{c \mathbf{k}}}+\mathrm{c.c}
\end{aligned}
$$

and solve the linear system of equations,

$X \cdot \alpha=t$

where the $t_{n}$ vector components are [13]: 


$$
\begin{aligned}
t_{n}= & \sum_{v} \sum_{c} \sum_{\mathbf{k}} \int \mathrm{d} \mathbf{r}_{1} f_{n}\left(\mathbf{r}_{1}\right) \\
& \times \int \mathrm{d} \mathbf{r}_{2} \frac{\psi_{v \mathbf{k}}^{*}\left(\mathbf{r}_{2}\right) \psi_{c \mathbf{k}}\left(\mathbf{r}_{2}\right)}{\left|\mathbf{r}_{1}-\mathbf{r}_{2}\right|}\left\langle\psi_{v \mathbf{k}}\left|V_{x}^{\mathrm{NL}}\right| \psi_{c \mathbf{k}}\right\rangle
\end{aligned}
$$

$V_{x}^{\mathrm{NL}}$ is the non-local exchange potential and $c$ represents an unoccupied monoelectronic state.

The two methods, KLI and EXX, presented here have satisfactory solutions to the OEP integral equation and a well-defined expression for the exchange (or exchange-correlation in the KLI case) potential. But in the KLI method, the substitution of the energy differences in Eq. (10) for a constant is a significant approximation for correctly describing band energies in solids. As we are interested in studying semiconductor materials we have chosen to develop and implement the EXX method, but using a version for a localised basis set instead of plane waves, compatible with the SIESTA program.

\section{Implementation of the EXX method within the SIESTA code}

In this section we present and discuss the integrals involved in the calculation of the exchange potential which can be solved within the SIESTA code. Our implementation is carried out for solids, and we take advantage of the translational symmetry between the cells in the solid.

The calculation of the exchange potential is made in three steps. First at all, we get the representation matrix elements for the linear response operator: Eq. (19). Then, we obtain the representation elements of the vector $t$ : Eq. (22). After that we solve the system of equations stated in Eq. (21) and we get the coefficients to represent the exchange density in the chosen basis set. Finally, Eq. (18) is solved in order to obtain the exchange potential.

Firstly the calculation of the integrals involved in the representation of the linear response operator, Eq. (19), must be made.

We start rewriting the definition for the linear response operator, thus,

$$
\begin{aligned}
X_{i j}= & \int \mathrm{d} \mathbf{r}_{1} f_{i}\left(\mathbf{r}_{1}\right) \int \frac{\mathrm{d} \mathbf{r}_{2}}{\left|\mathbf{r}_{1}-\mathbf{r}_{2}\right|} \int \mathrm{d} \mathbf{r}_{3} X\left(\mathbf{r}_{2}, \mathbf{r}_{3}\right) \\
& \times \int \frac{\mathrm{d} \mathbf{r}_{4}}{\left|\mathbf{r}_{3}-\mathbf{r}_{4}\right|} f_{j}\left(\mathbf{r}_{4}\right) \\
= & \int \mathrm{d} \mathbf{r}_{1} f_{i}\left(\mathbf{r}_{1}\right) \int \frac{\mathrm{d} \mathbf{r}_{2}}{\left|\mathbf{r}_{1}-\mathbf{r}_{2}\right|} \int \mathrm{d} \mathbf{r}_{3} X\left(\mathbf{r}_{2}, \mathbf{r}_{3}\right) v_{j}\left(\mathbf{r}_{3}\right) \\
= & \int \mathrm{d} \mathbf{r}_{1} f_{i}\left(\mathbf{r}_{1}\right) \int \frac{\mathrm{d} \mathbf{r}_{2}}{\left|\mathbf{r}_{1}-\mathbf{r}_{2}\right|} \zeta_{j}\left(\mathbf{r}_{2}\right) \\
= & \int \mathrm{d} \mathbf{r}_{1} f_{i}\left(\mathbf{r}_{1}\right) \mu_{j}\left(\mathbf{r}_{1}\right)
\end{aligned}
$$

where we define the following arrays,

$\mu_{j}\left(\mathbf{r}_{1}\right)=\int \mathrm{d} \mathbf{r}_{2} \frac{\zeta_{j}\left(\mathbf{r}_{2}\right)}{\left|\mathbf{r}_{1}-\mathbf{r}_{2}\right|}$

$\zeta_{j}\left(\mathbf{r}_{2}\right)=\int \mathrm{d} \mathbf{r}_{3} v_{j}\left(\mathbf{r}_{3}\right) X\left(\mathbf{r}_{2}, \mathbf{r}_{3}\right)$

$v_{j}\left(\mathbf{r}_{3}\right)=\int \mathrm{d} \mathbf{r}_{4} \frac{f_{j}\left(\mathbf{r}_{4}\right)}{\left|\mathbf{r}_{3}-\mathbf{r}_{4}\right|}$

$v_{j}\left(\mathbf{r}_{3}\right)$ is the array that contains the potential integrals coming from the basis functions to be used. The matrix element of the linear response operator is obtained as the integral of the array $\mu_{j}\left(\mathbf{r}_{1}\right)$ multiplied by basis function $f_{i}\left(\mathbf{r}_{1}\right)$, as is shown in Eq. (23).

In principle, any form of the basis set could be used to represent the exchange density. From a practical point of view, we have chosen the following basis that has the translational symmetry of the solid:

$f_{j}\left(\mathbf{r}_{4}\right)=\sum_{\mathbf{R}} \mathrm{e}^{i \mathbf{k R}} h_{j}\left(\mathbf{r}_{4}-\mathbf{R}-\mathbf{a}_{j}\right)$

where $\mathbf{R}$ is a vector connecting two different cells in the solid and its expression in terms of the unit cell vectors of the solid, $\left[\mathbf{b}_{1}, \mathbf{b}_{2}, \mathbf{b}_{3}\right]$, is: $\mathbf{R}=n_{1} \mathbf{b}_{1}+$ $n_{2} \mathbf{b}_{2}+n_{3} \mathbf{b}_{3} . \mathbf{a}_{j}$ represents the position where the function $h_{j}(\mathbf{r})$ is centered in the unit cell.

The $v_{j}\left(\mathbf{r}_{3}\right)$ vector components are obtained as the potential of each different basis function $f_{j}\left(\mathbf{r}_{4}\right)$ in all space. With this function $f_{j}\left(\mathbf{r}_{4}\right)$, expressed in terms of the $h_{j}\left(\mathbf{r}_{4}-\mathbf{R}-\mathbf{a}_{j}\right)$, Eq. (26) becomes,

$v_{j}\left(\mathbf{r}_{3}\right)=\sum_{\mathbf{R}} \mathrm{e}^{\mathbf{i k R} \hat{v}_{j}}\left(\mathbf{r}_{3}-\mathbf{R}-\mathbf{a}_{j}\right)$ 
where the components of the vector $\hat{v}_{j}\left(\mathbf{r}_{3}-\mathbf{R}-\mathbf{a}_{j}\right)$ are defined as,

$\hat{v}_{j}\left(\mathbf{r}_{3}-\mathbf{R}-\mathbf{a}_{j}\right)=\int \mathrm{d} \mathbf{r}_{4} \frac{h_{j}\left(\mathbf{r}_{4}-\mathbf{R}-\mathbf{a}_{j}\right)}{\left|\mathbf{r}_{3}-\mathbf{r}_{4}\right|}$

This integral has to be solved numerically for each function $h_{j}\left(\mathbf{r}_{4}-\mathbf{R}-\mathbf{a}_{j}\right)$ within the supercell. Nevertheless, as we have chosen Slater functions centered on the different atomic positions to represent the exchange density, this integral is solved analytically [31] and we directly use the expression of the potential associated with each one of the representation functions.

The next step consists of the integration of the resulting array components $v_{j}\left(\mathbf{r}_{3}\right)$ multiplied by the linear response operator $X\left(\mathbf{r}_{2}, \mathbf{r}_{3}\right)$ to obtain the $\zeta_{j}\left(\mathbf{r}_{2}\right)$ vector components.

As we have broken down the array $v_{j}\left(\mathbf{r}_{3}\right)$ into its sum of different components, each one of the integrals appearing in Eq. (25) is divided into a sum of integrals,

$$
\begin{aligned}
\zeta_{j}\left(\mathbf{r}_{2}\right) & =\int \mathrm{d} \mathbf{r}_{3} v_{j}\left(\mathbf{r}_{3}-\mathbf{R}-\mathbf{a}_{j}\right) X\left(\mathbf{r}_{2}, \mathbf{r}_{3}\right) \\
& =N \sum_{\mathbf{R}} \mathrm{e}^{\mathrm{ikR}} \int_{\text {onecell }} \mathrm{d} \mathbf{r}_{3} \widehat{v}_{j}\left(\mathbf{r}_{3}-\mathbf{R}-\mathbf{a}_{j}\right) X\left(\mathbf{r}_{2}, \mathbf{r}_{3}\right)
\end{aligned}
$$

where $N$ represents the total number of cells involved in the calculation. To solve the integrals, the potentials must be interpolated from a stored table of values and its angular part added in each different point of the space. For the values of the $X\left(\mathbf{r}_{2}, \mathbf{r}_{3}\right)$ operator, the following translation property is used,

$X\left(\mathbf{r}_{2}+\mathbf{R}, \mathbf{r}_{3}+\mathbf{R}^{\prime}\right)=X\left(\mathbf{r}_{2}, \mathbf{r}_{3}\right)$

where $\mathbf{R}^{\prime}$ is also a translation vector of the lattice.

Finally we total all the used cells and add the phase shifts, $\mathrm{e}^{\mathrm{ikR}}$ in order to obtain the $\zeta_{j}\left(\mathbf{r}_{2}\right)$ vector components, as is shown in Eq. (30).

The $\zeta_{j}\left(\mathbf{r}_{2}\right)$ array components still retain the translational symmetry of the solid with a phase shift of $\mathrm{e}^{\mathrm{ikR}}$ between two cells connected by the $\mathbf{R}$ vector. Due to this fact, it is only necessary to store the value of this array in the points lying within the unit cell.
The next step is to solve numerically the integral shown in Eq. (24), and store the result in the array $\mu_{j}\left(\mathbf{r}_{1}\right)$ components. This array $\mu_{j}\left(\mathbf{r}_{1}\right)$ still holds the translational symmetry with the phase shift $e^{i k R}$, and we have only stored its values for the points lying within the unit cell and interpolated as needed.

Finally, the linear response matrix elements is obtained using the relation $X_{i j}=\int \mathrm{d} \mathbf{r}_{1} f_{i}\left(\mathbf{r}_{1}\right) \mu_{j}\left(\mathbf{r}_{1}\right)$, where the integral is solved numerically.

The following step of the calculation is to obtain of the $t_{n}$ vector elements in Eq. (22). This calculation can be made in two parts. First of all, the integrals incorporating the $V_{x}^{\mathrm{NL}}\left(\mathbf{r}, \mathbf{r}^{\prime}\right)$ operator are obtained, and then the integral of the product of the two Kohn-Sham orbitals, $\psi_{v \mathbf{k}}^{*}\left(\mathbf{r}_{2}\right) \psi_{c \mathbf{k}}\left(\mathbf{r}_{2}\right)$, is solved.

In order to obtain the two electron integrals, we express the non-local exchange potential $V_{x}^{\mathrm{NL}}$ in terms of the Kohn-Sham orbitals,

$V_{x}^{\mathrm{NL}}\left(\mathbf{r}, \mathbf{r}^{\prime}\right)=\sum_{v^{\prime} \mathbf{q}} \frac{\psi_{v^{\prime} \mathbf{q}}(\mathbf{r}) \psi_{v^{\prime} \mathbf{q}}\left(\mathbf{r}^{\prime}\right)}{\left|\mathbf{r}-\mathbf{r}^{\prime}\right|}$

that when acting on the occupied KS-orbitals $\psi_{v \mathbf{k}}(\mathbf{r})$ the result becomes,

$J_{v \mathbf{k}}(\mathbf{r})=\sum_{v^{\prime} \mathbf{q}} \psi_{v^{\prime} \mathbf{q}}(\mathbf{r}) n_{v v^{\prime} \mathbf{q} \mathbf{k}}(\mathbf{r})$

where we define,

$n_{v v^{\prime} \mathbf{q} \mathbf{k}}(\mathbf{r})=\int \mathrm{d} \mathbf{r}^{\prime} \frac{\psi_{v^{\prime} \mathbf{q}}^{*}\left(\mathbf{r}^{\prime}\right) \psi_{v \mathbf{k}}\left(\mathbf{r}^{\prime}\right)}{\left|\mathbf{r}-\mathbf{r}^{\prime}\right|}$

The product of the two Kohn-Sham orbitals within the integral has a phase shift $\mathrm{e}^{\mathrm{i}(\mathbf{k}-\mathbf{q}) \mathbf{R}}$ that connects two cells by the translation vector $\mathbf{R}$. Then, we evaluate this integral using,

$$
\begin{aligned}
n_{v v^{\prime} \mathbf{q} \mathbf{k}}(\mathbf{r}) & =\sum_{\mathbf{R}} \int_{\text {cell }} \mathrm{d} \mathbf{r}^{\prime} \frac{\mathrm{e}^{-\mathrm{i} \mathbf{q} \mathbf{R}} \psi_{v^{\prime} \mathbf{q}}^{*}\left(\mathbf{r}^{\prime}\right) \mathrm{e}^{\mathrm{ikR} \mathbf{R}} \psi_{v \mathbf{k}}\left(\mathbf{r}^{\prime}\right)}{\left|\mathbf{r}-\mathbf{r}^{\prime}+\mathbf{R}\right|} \\
& =\sum_{\mathbf{R}} \mathrm{e}^{\mathrm{i}(\mathbf{k}-\mathbf{q}) \mathbf{R}} \int \mathrm{d} \mathbf{r}^{\prime} \frac{\psi_{v^{\prime} \mathbf{q}}^{*}\left(\mathbf{r}^{\prime}\right) \psi_{c \mathbf{k}}\left(\mathbf{r}^{\prime}\right)}{\left|\mathbf{r}-\left(\mathbf{r}^{\prime}-\mathbf{R}\right)\right|}
\end{aligned}
$$

The results are stored in the array $J_{v \mathbf{k}}(\mathbf{r})$ which depends only on two indices, the $\mathbf{k}$ vector and the occupied state label $v$.

The $J_{v \mathbf{k}}(\mathbf{r})$ array has the same translational properties as the Kohn-Sham orbitals, so we need 
only to store it in the points lying within the unit cell.

Finally, the $J_{v \mathbf{k}}(\mathbf{r})$ components multiplied by the occupied Kohn-Sham orbitals (i.e. we have to solve $\left.\int \mathrm{d} \mathbf{r} \psi_{c \mathbf{k}}^{*}(\mathbf{r}) J_{v \mathbf{k}}(\mathbf{r})\right)$, must be integrated. Because the integrand never goes to zero, the integrals can be solved if the Kohn-Sham orbital is broken down into a basis set representation, made up of atomic centered functions, $\chi_{j}(\mathbf{r})$, that are different from those used to represent the exchange density. Thus we obtain the integral,

$$
\begin{aligned}
\int \mathrm{d} \mathbf{r} \psi_{c \mathbf{k}}(\mathbf{r}) J_{v \mathbf{k}}(\mathbf{r})= & \sum_{\mathbf{R}} \mathrm{e}^{\mathrm{ik \mathbf {R }}} \sum_{j} d_{j c}(\mathbf{k}) \\
& \times \int \mathrm{d} \mathbf{r} \chi_{j}\left(\mathbf{r}-\mathbf{R}-\mathbf{a}_{j}\right) J_{v \mathbf{k}}(\mathbf{r})
\end{aligned}
$$

where $d_{j c}(\mathbf{k})$ are the representation coefficients of the Kohn-Sham orbitals in the $\chi(\mathbf{r})$ basis set.

The last integral converges, because the functions used to represent the Kohn-Sham orbitals within the SIESTA [29] code, are zero beyond a cutoff radius.

The last integral is then solved as,

$$
\begin{aligned}
& \int_{\text {allspace }} \mathrm{d} \mathbf{r} \chi_{j}\left(\mathbf{r}-\mathbf{R}-\mathbf{a}_{j}\right) J_{c \mathbf{k}}(\mathbf{r}) \\
& =\sum_{\mathbf{R}^{\prime}} \int_{\text {cell }} \mathrm{d} \chi_{j}\left(\mathbf{r}-\mathbf{R}-\mathbf{R}^{\prime}-\mathbf{a}_{j}\right) J_{c \mathbf{k}}\left(\mathbf{r}-\mathbf{R}^{\prime}\right)
\end{aligned}
$$

The functions $J_{c \mathbf{k}}(\mathbf{r})$ within this integral are obtained from their stored values in the unit cell, and the functions $\chi_{j}(\mathbf{r})$ have to be obtained in each different point in space.

To obtain the $t_{n}$ vector components, we have to solve the integrals in $\mathbf{r}_{1}$ and in $\mathbf{r}_{2}$ as appear in Eq. (22). To obtain the potential corresponding to each pair of functions, $\psi_{v \mathbf{k}}^{*}\left(\mathbf{r}_{2}\right) \psi_{c \mathbf{k}}\left(\mathbf{r}_{2}\right)$ throughout the space, we only store it within the unit cell, due to the translation property of the Kohn-Sham orbitals product with the same $\mathbf{k}$. The result of this integral is stored in the array

$r_{v c \mathbf{k}}\left(\mathbf{r}_{1}\right)=\int \mathrm{d} \mathbf{r}_{2} \frac{\psi_{v \mathbf{k}}^{*}\left(\mathbf{r}_{2}\right) \psi_{c \mathbf{k}}\left(\mathbf{r}_{2}\right)}{\left|\mathbf{r}_{1}-\mathbf{r}_{2}\right|}$

Finally the $t_{n}$ vector components are calculated as: $t_{n}=\sum_{v} \sum_{c} \sum_{\mathbf{k}} \int \mathrm{d} \mathbf{r}_{1} f_{n}\left(\mathbf{r}_{1}\right) r_{v c \mathbf{k}}\left(\mathbf{r}_{1}\right)$

Due to the properties of the function inside the integral, we can break down the integral over cells as,

$t_{n}=\sum_{v} \sum_{c} \sum_{\mathbf{k}} \sum_{\mathbf{R}} \int \mathrm{d} \mathbf{r}_{1} f_{n}\left(\mathbf{r}_{1}+\mathbf{R}\right) r_{v c \mathbf{k}}\left(\mathbf{r}_{1}\right)$

The vector components $r_{v c k}\left(\mathbf{r}_{1}\right)$ are obtained from the values within the unit cell, and the value of each basis function is interpolated as done in the final step of the representation of the linear response operator.

To obtain the expansion coefficients for the exchange density, the linear set of equations shown in Eq. (21) must be solved using the $t_{n}$ components and the elements of the representation matrix for the linear response operator $X_{i j}$.

The exchange potential is finally obtained by the direct integration of the exchange density as shown in Eq. (18).

\section{Computational details}

Here we present the computational details used in the EXX calculations within the efficient SIESTA program. SIESTA is a code based in the use of a linear combination of localized finite-range numerical pseudoatomic wave functions [32]. It usually uses norm conserving pseudopotentials in their non-local form, i.e. it uses one different radial part for each different value of the quantum number $l$ included in the pseudopotential [33-35]. These pseudopotentials are generated using the Troullier-Martins parameterization [36], and in SIESTA they are transformed into their non-local form using Kleinmann-Bylander projectors [3740]. The cutoff radii used in the pseudopotential generation are shown in Table 1.

The different pseudopotential components have been developed using the atom code. This code has been modified and we have generated these pseudopotentials using the EXX [41] exchange term instead of the LDA or GGA approximations. The SIESTA calculation is made in the usual way, but the exchange potential is obtained by using the 
Table 1

Cutoff radii used for the generation of the LDA and EXX pseudopotentials, generated using the Troullier-Martins scheme, in a.u

\begin{tabular}{lllll}
\hline & $\mathrm{s}$ & $\mathrm{p}$ & $\mathrm{d}$ & $\mathrm{f}$ \\
\hline $\mathrm{Si}(3 \mathrm{~s}, 3 \mathrm{p}, 3 \mathrm{~d}, 4 \mathrm{f})$ & 1.90 & 1.90 & 1.90 & 1.90 \\
$\mathrm{Ge}(4 \mathrm{~s}, 4 \mathrm{p}, 4 \mathrm{~d}, 4 \mathrm{f})$ & 2.00 & 2.00 & 2.20 & 2.20 \\
$\mathrm{C}(2 \mathrm{~s}, 2 \mathrm{p}, 3 \mathrm{~d}, 4 \mathrm{f})$ & 1.25 & 1.25 & 1.25 & 1.25 \\
$\mathrm{Ga}(4 \mathrm{~s}, 4 \mathrm{p}, 4 \mathrm{~d}, 4 \mathrm{f})$ & 2.00 & 2.00 & 2.80 & 2.80 \\
$\mathrm{As}(3 \mathrm{~s}, 3 \mathrm{p}, 3 \mathrm{~d}, 4 \mathrm{f})$ & 2.55 & 2.55 & 2.55 & 2.55 \\
$\mathrm{Al}(4 \mathrm{~s}, 4 \mathrm{p}, 4 \mathrm{~d}, 4 \mathrm{f})$ & 2.30 & 2.30 & 2.30 & 2.30 \\
$\mathrm{P}(3 \mathrm{~s}, 3 \mathrm{p}, 3 \mathrm{~d}, 4 \mathrm{f})$ & 1.55 & 1.55 & 1.80 & 1.80 \\
\hline
\end{tabular}

EXX method in each cycle of the SCF convergence procedure. In the calculation we have used mesh and $k$-grid cutoffs large enough to ensure the total convergence in the integrals.

As said in the previous section we have used two different basis of functions, one for the representation of the Kohn-Sham orbitals and another basis used to represent the exchange density. The basis for the orbitals is automatically generated by the SIESTA code, and we only change the basis size in order to get additional information.
The basis used to represent the exchange potential is made up of a set of Slater functions large enough to consider a complete basis set representation.

The new added integrals are solved in real or reciprocal spaces, depending on the integral. In both cases SIESTA's philosophy has been followed. Thus the real space integrals have been solved using the Simpson rule [42], and the reciprocal space integrals have been solved using the fast Fourier transform method [42]. In the previous section we have already discussed the particular solution of each one of the added integrals. The inversion of the representation matrix for the linear response operator has been carried out using the LU inversion algorithm [42].

\section{Results}

\subsection{Band gaps and bandwidths}

In this section we present the results obtained when the EXX formalism is used for the study of

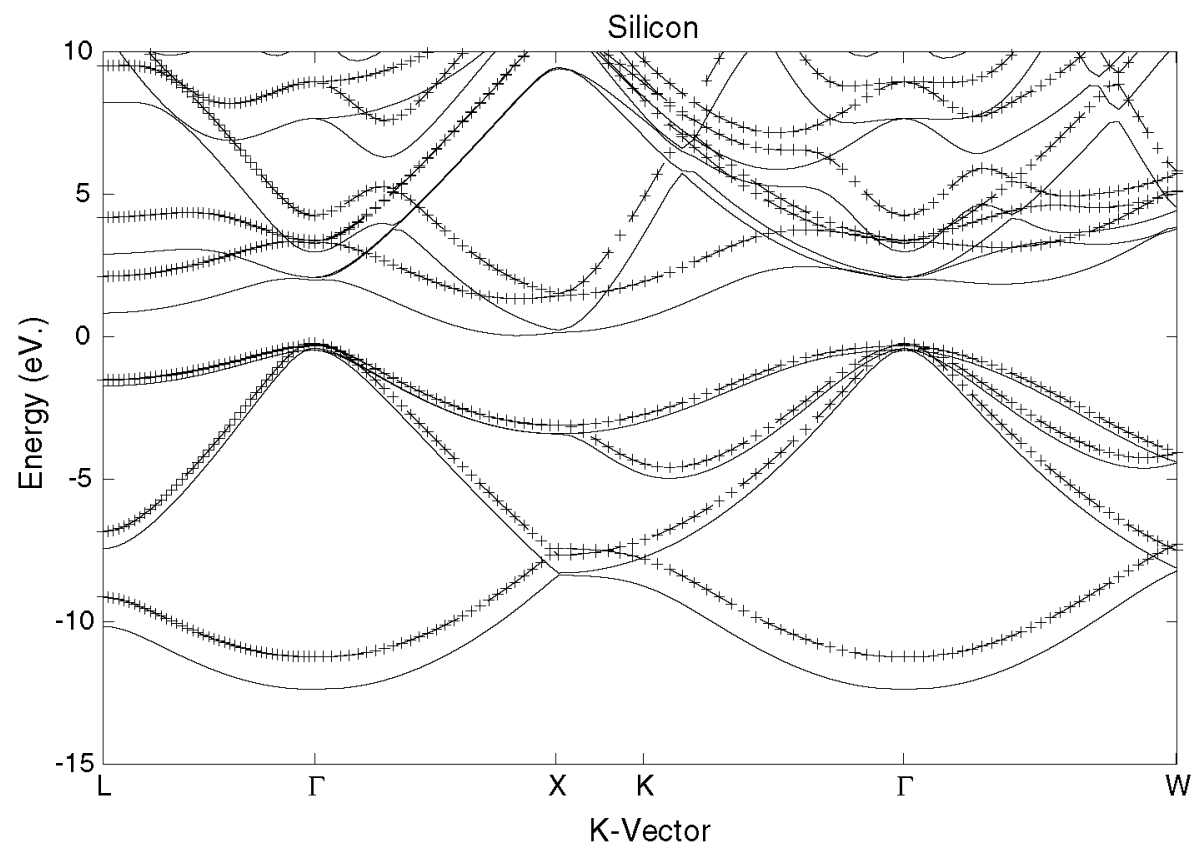

Fig. 1. Band structure for Si calculated within the LDA (solid lines) and EXX (crossed lines) methods. The bands are displayed along the most significant directions in the diamond structure. 
the band gaps and the bandwidths of the III-V semiconductors. Figs. 1 and 2 show the dispersion band diagrams obtained for the silicon and the gallium arsenide semiconductors. As can be seen, the effect of the EXX potential yields a higher localization of the bands with respect to the LDA or GGA results. The valence bandwidth is lowered and the conduction band is less bounded resulting in a enlargement of the band-gap width. This is due mainly to the non-self-interaction character of the EXX potential.

The comparison of the dispersion band diagrams for several semiconductors show a significant $k$-dependence of the EXX energies values. This fact reflects the $k$-dependence of the exchange potential [14]. Also the shape of the EXX band structures obtained compared with band-structure forms found in literature [14] and with experimental results agree very well.

In Tables 2-4 we present the different band gaps for several semiconductors in a number of high symmetry points ( $L, \Gamma$ and $X$ points respectively) and, in Table 5, the minimum band gap values, for the zincblende unit cell structure. These band gaps are always greater than those calculated with the LDA exchange functional and with other similar ones found in literature. The EXX values are in good agreement with experimental values, as well as with those obtained with the plane wave EXX calculations. It is worth noting that in spite of the good agreement results, the absolute errors between experimental and EXX could be attributed to the fact that correlation functionals also needs to be "conveniently" modified.

Table 6 shows the variation of the minimum energy gap value for several semiconductors according to the representation basis set of the Kohn-Sham orbitals. The most simple possible description of the Kohn-Sham orbitals, the simple $\zeta$ basis set (SZ), is made using only one numerical basis function to describe the KS-orbital. This could be improved by the double $\zeta$ (DZ) basis set, where two numerical basis functions are used to describe each orbital. Finally the DZP basis set is obtained by adding one polarization function per orbital to the DZ basis set [29].

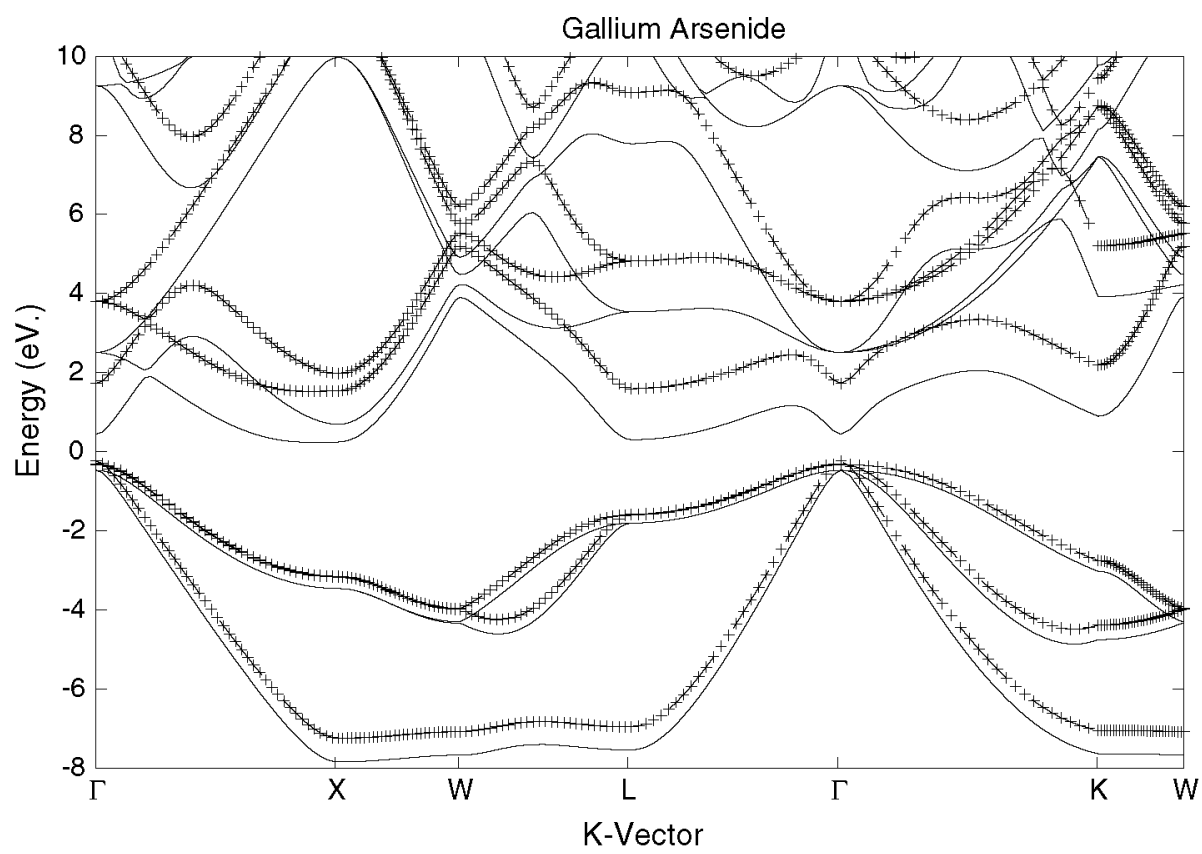

Fig. 2. Band structure for GaAs calculated within the LDA (solid lines) and EXX (crossed lines) methods. The bands are displayed along the most significant directions in the zincblende (ZB) structure. 
Table 2

Band gap values obtained in the $\mathrm{L}$ point of the Brillouin zone (BZ), using the LDA and the EXX approximations for the exchange term and a DZP basis set

\begin{tabular}{lllll}
\hline & LDA & EXX & EXX $^{\mathrm{a}}$ & Experim. \\
\hline $\mathrm{Si}$ & 1.43 & 2.63 & 2.36 & $2.40^{\mathrm{b}}$ \\
$\mathrm{Ge}$ & 0.13 & 1.08 & 1.01 & $0.84^{\circ}$ \\
$\mathrm{C}$ & 8.43 & 9.18 & 9.18 & \\
$\mathrm{GaAs}$ & 0.89 & 1.93 & 1.93 & $1.85^{\mathrm{d}}$ \\
$\mathrm{AlAs}$ & 2.03 & 2.95 & 2.99 & $2.54^{\mathrm{c}}$ \\
$\mathrm{SiC}$ & 5.36 & 6.25 & 6.30 & \\
$\mathrm{GaP}$ & 7.16 & 8.60 & & \\
\hline
\end{tabular}

The last column contains experimental values, in $\mathrm{eV}$.

${ }^{\text {a }}$ Ref. [14].

${ }^{\mathrm{b}}$ Ref. [44].

${ }^{c}$ Ref. [45].

${ }^{\mathrm{d}}$ Ref. [46].

Table 3

Band gap values in the $\Gamma$ point of the zincblende Brillouin zone (BZ)

\begin{tabular}{lrlll}
\hline & LDA & EXX & EXX $^{\mathrm{a}}$ & Experim. \\
\hline $\mathrm{Si}$ & 0.65 & 3.49 & $3.26-3.46$ & $3.34^{\mathrm{a}}$ \\
$\mathrm{Ge}$ & -0.09 & 1.31 & 1.28 & $1.00^{\mathrm{b}}$ \\
$\mathrm{C}$ & 5.56 & 6.32 & 6.28 & $7.30^{\mathrm{a}}$ \\
$\mathrm{GaAs}$ & 0.32 & 1.90 & 1.82 & $1.63^{\mathrm{c}}$ \\
$\mathrm{AlAs}$ & 1.84 & 3.25 & 3.20 & $3.11^{\mathrm{b}}$ \\
$\mathrm{SiC}$ & 6.33 & 7.45 & 7.37 & $7.59^{\mathrm{d}}$ \\
$\mathrm{GaP}$ & 4.20 & 5.69 & & \\
\hline
\end{tabular}

Same legend as Table 2.

Table 4

Band gap values in the $\mathrm{X}$ point of the zincblende Brillouin zone (BZ)

\begin{tabular}{lllll}
\hline & LDA & EXX & EXX $^{\mathrm{a}}$ & Experim. \\
\hline $\mathrm{Si}$ & 0.67 & 1.45 & 1.43 & $1.25^{\mathrm{b}}$ \\
$\mathrm{Ge}$ & 0.75 & 1.38 & 1.34 & $1.30^{\circ}$ \\
$\mathrm{C}$ & 4.78 & 6.32 & 5.43 & \\
$\mathrm{GaAs}$ & 1.41 & 1.84 & 2.15 & $2.18^{\mathrm{d}}$ \\
$\mathrm{AlAs}$ & 1.37 & 2.28 & 2.26 & $2.24^{\mathrm{c}}$ \\
$\mathrm{SiC}$ & 1.36 & 2.58 & 2.52 & $2.39^{\mathrm{b}}$ \\
$\mathrm{GaP}$ & 3.24 & 2.36 & & \\
\hline
\end{tabular}

Same legend as Table 2.

\subsection{Exchange and total energies}

The introduction of the EXX term within the Kohn-Sham potential modifies both the total and
Table 5

Minimum band gap (eV) values for several semiconductors using the LDA and EXX approximations for the exchange term, compared with experimental values

\begin{tabular}{llll}
\hline & LDA & EXX & Experim. \\
\hline $\mathrm{Si}$ & 1.01 & 1.30 & $1.12^{\mathrm{a}}$ \\
$\mathrm{Ge}$ & 0.19 & 0.74 & $0.70^{\mathrm{b}}$ \\
$\mathrm{C}$ & 4.50 & 5.44 & $5.46-5.60^{\mathrm{c}}$ \\
$\mathrm{GaAs}$ & 0.80 & 1.49 & $1.42^{\mathrm{c}}$ \\
$\mathrm{AlAs}$ & 2.13 & 2.36 & $2.24^{\mathrm{b}}$ \\
$\mathrm{SiC}$ & 1.78 & 2.48 & $2.36^{\mathrm{a}}$ \\
$\mathrm{GaP}$ & 1.56 & 2.62 & $2.26^{\mathrm{a}}$ \\
\hline
\end{tabular}

${ }^{\mathrm{a}}$ Ref. [14].

${ }^{\mathrm{b}}$ Ref. [43]

${ }^{\mathrm{c}}$ Ref. [44].

Table 6

Minimum band gap $(\mathrm{eV})$ for several qualities of basis sets used to represent the KS-orbitals, for the LDA and EXX exchange functionals

\begin{tabular}{|c|c|c|c|c|c|c|}
\hline & \multicolumn{2}{|l|}{ SZ } & \multicolumn{2}{|l|}{$\mathrm{DZ}$} & \multicolumn{2}{|l|}{ DZP } \\
\hline & $\mathrm{LDA}$ & EXX & LDA & EXX & LDA & $\mathrm{EXX}$ \\
\hline $\mathrm{Si}$ & 1.89 & 1.91 & 1.52 & 1.67 & 1.01 & 1.30 \\
\hline $\mathrm{Ge}$ & 0.31 & 1.05 & 0.37 & 0.89 & 0.19 & 0.74 \\
\hline $\mathrm{C}$ & 6.05 & 6.47 & 5.66 & 6.17 & 4.50 & 5.44 \\
\hline GaAs & 0.98 & 1.90 & 0.99 & 1.93 & 0.80 & 1.49 \\
\hline AlAs & 2.40 & 2.54 & 2.20 & 2.40 & 2.13 & 2.36 \\
\hline GaP & 1.03 & 2.88 & 1.06 & 2.60 & 1.56 & 2.62 \\
\hline $\mathrm{SiC}$ & 4.69 & 4.80 & 4.28 & 4.60 & 1.78 & 2.48 \\
\hline
\end{tabular}

The values of the used mesh cutoff ensure the convergence of all integrals in all calculations.

exchange energy values. In Table 7 we compare the values obtained for the exchange energy using the LDA and the EXX approximations to represent the exchange term. We also present the results according to the three different quality basis sets used, simple zeta (SZ), double zeta (DZ) and double zeta with a polarization function basis set (DZP). As shown in the table, as the basis gets bigger, the total and exchange energies lower. This reflects the incompleteness of the $\mathrm{SZ}$ and DZ basis. Nevertheless because our purpose is to study the effect on the exchange and total energies, when the EXX approximation for the exchange is used instead of the LDA, we show that the introduction of the EXX exchange term lowers the exchange energy with respect to the LDA results in all cases. 
Table 7

Exchange energy (eV) as a function of different basis set used for the LDA and EXX functionals

\begin{tabular}{|c|c|c|c|c|c|c|}
\hline & \multicolumn{3}{|l|}{ LDA } & \multicolumn{3}{|l|}{ EXX } \\
\hline & $\mathrm{SZ}$ & $\mathrm{DZ}$ & DZP & $\mathrm{SZ}$ & $\mathrm{DZ}$ & $\mathrm{SZP}$ \\
\hline $\mathrm{Si}$ & -64.42 & -65.06 & -65.29 & -64.47 & -65.08 & -65.31 \\
\hline $\mathrm{Ge}$ & -61.30 & -62.10 & -62.29 & -61.94 & -62.31 & -62.81 \\
\hline $\mathrm{C}$ & -94.26 & -95.02 & -95.18 & -95.15 & -95.87 & -95.90 \\
\hline GaAs & -147.75 & -151.99 & -152.08 & -147.96 & -152.19 & -152.21 \\
\hline AlAs & -116.90 & -117.69 & -117.71 & -116.95 & -117.74 & -117.81 \\
\hline $\mathrm{GaP}$ & -95.20 & -96.17 & -96.65 & -95.61 & -96.39 & -96.88 \\
\hline $\mathrm{SiC}$ & -82.29 & -82.51 & -82.76 & -82.65 & -82.71 & -82.99 \\
\hline
\end{tabular}

Table 8

Total energy (eV) according to the basis set quality, using the LDA and the EXX functionals

\begin{tabular}{|c|c|c|c|c|c|c|}
\hline & \multicolumn{3}{|l|}{ LDA } & \multicolumn{3}{|c|}{$\mathrm{EXX}$} \\
\hline & $\mathrm{SZ}$ & $\mathrm{DZ}$ & DZP & $\mathrm{SZ}$ & $\mathrm{DZ}$ & DZP \\
\hline $\mathrm{Si}$ & \multicolumn{3}{|c|}{$-213.45-214.02-214.86$} & \multicolumn{3}{|c|}{$-214.24-214.81-214.97$} \\
\hline $\mathrm{Ge}$ & -214.62 & -215.54 & -216.32 & \multicolumn{3}{|c|}{$-216.20-216.51-217.17$} \\
\hline $\mathrm{C}$ & -309.39 & -310.46 & -310.90 & \multicolumn{3}{|c|}{$-310.14-310.78-311.40$} \\
\hline GaAs & -305.02 & -307.92 & -308.98 & \multicolumn{3}{|c|}{$-305.84-307.99-308.69$} \\
\hline AlAs & -274.96 & -275.42 & -276.06 & \multicolumn{3}{|c|}{$-274.98-275.66-276.90$} \\
\hline $\mathrm{GaP}$ & -265.88 & -266.49 & -267.99 & \multicolumn{3}{|c|}{$-266.13-266.88-268.08$} \\
\hline $\mathrm{SiC}$ & -265.31 & -265.39 & -265.37 & \multicolumn{3}{|c|}{$-265.80-265.86-265.92$} \\
\hline
\end{tabular}

Table 8 shows how the total energy of the system is affected by the use of the EXX exchange functional instead of the LDA one. This effect is similar to the effect on the exchange energy: the total energy is also lowered when the EXX functional is used. As has been said before, one of the effect of the use of the EXX potential is the self-interaction freeness. The main consequence of this effect causes in the Kohn-Sham states to be more bounded, and the eigenvalues of the Kohn-Sham energies to be lowered. This effect produces a more negative contribution to the band structure term to the total energy $[15,29]$.

\subsection{Atomic positions}

Another important factor to take into account when introducing the EXX exchange potential instead of the LDA exchange potential is in the total force on the atoms. For this reason we present results obtained in the relaxation of the po-
Table 9

Displacements in the zincblende unit cell two atoms positions for several semiconductors with respect to the theoretical position

\begin{tabular}{|c|c|c|c|c|c|c|}
\hline & \multicolumn{3}{|l|}{ LDA } & \multicolumn{3}{|l|}{ EXX } \\
\hline & $\mathrm{X}$ & $\mathrm{Y}$ & Z & $\mathrm{X}$ & $\mathrm{Y}$ & $\mathrm{Z}$ \\
\hline \multirow[t]{2}{*}{$\mathrm{Si}$} & 0.2935 & 0.2935 & 0.2935 & 0.3590 & 0.3583 & 0.3587 \\
\hline & -0.2935 & -0.2935 & -0.2935 & -0.1086 & $5-0.1061$ & -0.1060 \\
\hline \multirow[t]{2}{*}{$\mathrm{GaP}$} & 0.2109 & 0.2109 & 0.2109 & 0.2230 & 0.2230 & 0.2230 \\
\hline & -0.2117 & $7-0.2117$ & $7-0.2117$ & -0.2242 & $2-0.2242$ & -0.2242 \\
\hline \multirow[t]{2}{*}{ GaAs } & 0.2142 & 0.2142 & 0.2142 & 0.2145 & 0.2145 & 0.2145 \\
\hline & -0.2140 & -0.2140 & -0.2140 & -0.2139 & -0.2139 & 0.2139 \\
\hline \multirow[t]{2}{*}{$\mathrm{SiC}$} & 0.1889 & 0.1889 & 0.1889 & 0.1990 & 0.1990 & 0.1990 \\
\hline & -0.1760 & -0.1760 & -0.1760 & -0.1880 & -0.1880 & -0.1880 \\
\hline \multirow[t]{2}{*}{ AlAs } & 0.1614 & 0.1614 & 0.1614 & 0.1732 & 0.1732 & 0.1732 \\
\hline & -0.1614 & $4-0.1614$ & -0.1614 & -0.1731 & $1-0.1731$ & -0.1731 \\
\hline \multirow[t]{2}{*}{$\mathrm{C}$} & 0.2841 & 0.2841 & 0.2841 & 0.2990 & 0.2990 & 0.2990 \\
\hline & -0.2841 & -0.2841 & -0.2841 & -0.2990 & -0.2992 & -0.2991 \\
\hline
\end{tabular}

The values included in the table are in units of the lattice parameter, and have to be divided by a factor of 100 .

sitions of the unit cell atoms for $\mathrm{Si}$ and $\mathrm{C}$ and several III-V semiconductors.

Table 9 shows the values of the displacements in the zincblende unit cell atom positions for several semiconductors. These displacements represent the difference in the final position of the atoms with respect to the theoretical positions, expressed in terms of units of the lattice parameter, $(0,0,0)$, $(0.25,0.25,0.25)$ as input positions. The relaxation procedure was carried out within the conjugate gradient (CG) method and is finished when the total forces on the atoms become less than $40 \mathrm{meV} /$ $\AA^{3}$. The first three values within each row correspond with the displacements obtained in a calculation using the LDA approximation for the exchange term. The remaining three values correspond to the displacements obtained in a calculation using the EXX approximation for the exchange term. As can be seen in the table, the difference between the values corresponding to the LDA and the EXX cases are by no meaning significant.

\section{Conclusions}

In this paper we have presented the theoretical development and the implementation of the EXX 
exchange functional in the numerical LCAO DFT SIESTA code and the improvement in the description of electronic properties of semiconductors by means of the exact exchange method.

From the methodological point of view we have discussed how the implementation of the EXX formalism in SIESTA is carried out. In this aspect, the attention has been mainly focused on overcoming practical problems in coding the EXX exchange part of the potential within the SIESTA code framework, especially for the evaluation of large two-electron integrals which do not need to be calculated in the usual LDA or GGA approximations.

As is shown in previous section, the use of the EXX in a localized LCAO basis set scheme, give an accurate description of the band structure and the band gap values for semiconductors. As expected, EXX results agree better with the experimental values than the LDA results. Moreover, many other properties can also be calculated depending on the system studied, using the SIESTA code and the EXX formalism for the exchange part in the KS-potential. The application is the subject of further works.

\section{Acknowledgements}

We wish to thank Professor A. Görling for fruitful discussions during the early stages of this work. We are indebted to E. Artacho, P. Ordejón, D. Sanchez-Portal, J.M. Soler and A. García for providing us the SIESTA computational code. This work was partially supported by Spain's Plan Nacional de I $+\mathrm{D}$ program under reference TIC2000-1339-C02-02 and the European Commission contract ENK6 CT200 00310.

\section{References}

[1] R.G. Parr, W. Yang, Density Functional Theory, Oxford University Press, Oxford, 1989.

[2] P. Hohemberg, W. Kohn, Phys. Rev. B 136 (1964) 864.

[3] W. Kohn, L.J. Sham, Phys. Rev. A 140 (1965) 1133.

[4] J.P. Perdew, A. Zunger, Phys. Rev. B 23 (1981) 5048.

[5] A.D. Becke, Phys. Rev. A 140 (1988) 3098.

[6] J.P. Perdew, M. Levy, Phys. Rev. Lett. 51 (1993) 1884.
[7] J.P. Perdew, K. Burke, M. Ernzerhof; Phys. Rev. Lett. 77 (1996) 3865, 78 (1997) 1396.

[8] A. Görling, Phys. Rev. A 47 (1993) 2783.

[9] A. Görling, Phys. Rev. A 46 (1993) 5155.

[10] A. Görling, M. Levy, Int. J. Quantum Chem. Symp. 29 (1995) 93.

[11] A. Görling, M. Levy, Phys. Rev. B 47 (1993) 13105.

[12] A. Seidl, A. Görling, P. Vogl, J.A. Majewki, M. Levy, Phys. Rev. B 53 (1996) 3764.

[13] A. Görling, Phys. Rev. Lett. 83 (1999) 5459.

[14] M. Staëdelle, M. Moukara, J.A. Majewski, P. Vogl, A. Görling, Phys. Rev. B 59 (1999) 10031.

[15] M. Moukara, M. Staëdelle, J.A. Majewki, P. Vogl, A. Görling, J. Phys. Condens. Matter 12 (2000) 6783.

[16] J.B. Krieger, Y. Li, G.J. Iafrate, Phys. Lett. A 148 (1990) 470.

[17] J.B. Krieger, Y. Li, G.J. Iafrate, Phys. Rev A 45 (1992) 101.

[18] J.B. Krieger, Y. Li, G.J. Iafrate, Phys. Rev. A 47 (1993) 165.

[19] J.B. Krieger, Y. Li, G.J. Iafrate, Phys. Rev. A 46 (1992) 5453.

[20] T. Kotani, Phys. Rev. 50 (1994) 14816.

[21] T. Kotani, H. Akai, Phys. Rev. 52 (1995) 17153.

[22] T. Kotani, Phys. Rev. Lett. 74 (1995) 2989.

[23] T. Kotani, Phys. Rev. B 54 (1996) 16502.

[24] J. Garza, J.A. Nichols, D.A. Dixon, J. Chem. Phys. 112 (2000) 1150

[25] J. Garza, C.A. Fahlstrom, R. Vargas, J.A. Nichols, D.A. Dixon, Orbitals from Molecular Theories for Ionic Systems, in: Rev. Modern Quantum Chemistry: A celebration of: the contributions of R.G. Parr, K.D. Ed, Word Scientific, Singapore, 2002, pp. 1508-1536 (Chapter 50);

J. Garza, J.A. Nichols, D.A. Dixon, J. Chem. Phys. 112 (2000) 1150 ;

J. Garza, J.A. Nichols, D.A. Dixon, J. Chem. Phys. 112 (2000) 7880 ;

J. Garza, J.A. Nichols, D.A. Dixon, J. Chem. Phys. 113 (2001) 6029;

J. Garza, J.A. Nichols, D.A. Dixon, J. Chem. Phys. 114 (2001) 639;

S. Ivanov, S. Hirata, R.J. Barlett, J. Chem. Phys. 116 (2002) 1269.

[26] P. Wahnón, C. Tablero, Phys. Rev. B 65 (2002) 165115.

[27] C. Tablero, P. Wahnón, Appl. Phys. Lett. 82 (2003) 151.

[28] C. Tablero, A.J. Garcia, J.J. Fernández, P. Palacios, P. Wahnón, Comp. Mat. Sci. 27 (2003) 58.

[29] J.M. Soler, E. Artacho, J.D. Gale, A. Garcia, J. Junquera, P. Ordejon, D. Sanchez-Portal, J. Phys. Condens. Matter. 14 (2002) 2745;

E. Artacho et al., Phys. Status Solidi B 215 (1999) 809;

D. Sánchez-Portal et al., Int. J. Quantum Chem. 65 (1997) 453;

P. Ordejón et al., Phys. Rev. B 53 (1996) R10441.

[30] J.D. Talman, W.F. Shadwick, Phys. Rev. A 14 (1976) 36.

[31] M.A. Abrahowitz, I.S. Stegun, Mathematical Handbook of: Functions, Dover Publications Inc., New York, 1965, p. 117. 
[32] O.F. Sankey, D.J. Niklewki, Phys. Rev. B 41 (1989) 3979.

[33] G.B. Bachelet, D.R. Hamman, M. Schlüter, Phys. Rev. B 26 (1982) 4199.

[34] D. Vanderbilt, Phys. Rev. B 41 (1990) 7892.

[35] S. Fahny, X.W. Wang, S.G. Louie, Phys. Rev. Lett. 61 (1998) 1631.

[36] N. Trullier, J.L. Martins, Phys. Rev. B 43 (1993) 1494.

[37] D.M. Bylander, L. Kleinman, Phys. Rev. Lett. 74 (1995) 3660 .

[38] D.M. Bylander, L. Kleinman, Phys. Rev. B 52 (1995) 14566.

[39] D.M. Bylander, L. Kleinman, Phys. Rev. B 54 (1996) 7891.

[40] D.M. Bylander, L. Kleinman, Phys. Rev. B 55 (1997) 9432.

[41] J.J. Fernández, C. Tablero, P. Wahnón, Int. J. Quantum Chem. 91 (2003) 157.
[42] W.H. Press, B.P. Flanneery, S.A. Teukolsky, W.T. Vetterling, Numerical Recipes in FORTRAN, Cambridge University Press, Cambridge, 1999.

[43] J.E. Ortega, F.J. Himpsel, Phys. Rev. B 47 (1993) 2130.

[44] A. Mainwood, in: G. Davies (Ed.), Properties and Growth of diamonds, Electronic Materials Information Service, London, 1994, p. 3.

[45] G. Ramirez-Flores, H. Navarro-Contreras, A. LastrasMartinez, R.C. Powell, J.E. Greene, Phys. Rev. B (1994) 8433.

[46] M.E. Levinstein, S.L. Rumiantsev, M.S. Shur (Eds.), Handbook Series of Semiconductor Properties V. 1: Elementary Semiconductors and A3B5 Compounds Si, $\mathrm{Ge}, \mathrm{C}, \mathrm{GaAs}, \mathrm{GaP}, \mathrm{GaSb}$, InAs, InP, InSb, Word Science Publishers, New York, 1996. 